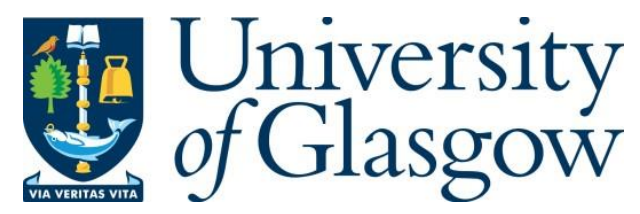

Di Campli San Vito, P., Brewster, S., Pollick, F., White, S., Skrypchuk, L. and Mouzakitis, A. (2018) Investigation of Thermal Stimuli for Lane Changes. In: AutomotiveUI' 18 10th International ACM Conference on Automotive User Interfaces and Interactive Vehicular Applications, Toronto, Canada, September 2325, 2018, pp. 43-52. ISBN 9781450359467.

There may be differences between this version and the published version. You are advised to consult the publisher's version if you wish to cite from it.

(C) The Authors 2018. This is the author's version of the work. It is posted here for your personal use. Not for redistribution. The definitive Version of Record was published in the Proceedings of the AutomotiveUI '18 10th International ACM Conference on Automotive User Interfaces and Interactive Vehicular Applications, Toronto, Canada, September 23-25, 2018, pp. 43-52. ISBN 9781450359467 https://doi.org/10.1145/3239060.3239062.

http://eprints.gla.ac.uk/166314/

Deposited on: 9 August 2018

Enlighten - Research publications by members of the University of Glasgow http://eprints.gla.ac.uk 


\section{Investigation of Thermal Stimuli for Lane Changes}

\author{
Patrizia Di Campli San Vito, \\ Stephen Brewster \\ Computing Science \\ University of Glasgow, United \\ Kingdom \\ p.di-campli-san- \\ vito.1@research.gla.ac.uk, \\ stephen.brewster@glasgow.ac.uk
}

\author{
Frank Pollick \\ Psychology \\ University of Glasgow, United \\ Kingdom \\ frank.pollick@glasgow.ac.uk
}

\author{
Stuart White, Lee Skrypchuk, \\ Alexander Mouzakitis \\ Jaguar Land Rover \\ Coventry, United Kingdom \\ swhite28@jaguarlandrover.com, \\ lskrypchuk@jaguarlandrover.com, \\ amouzaki- \\ tis@jaguarlandrover.com
}

\begin{abstract}
Haptic feedback has been widely studied for in-car interactions. However, most of this research has used vibrotactile cues. This paper presents two studies that examine novel thermal feedback for navigation during simulated driving for a lane change task. In the first, we compare the distraction and time differences of audio and thermal feedback. The results show that the presentation of thermal stimuli does not increase lane deviation, but the time needed to complete a lane change increased by 1.82 seconds. In the second study, the influence of variable changes of thermal stimuli on the lane change task performance was tested. We found that the same stimulus design for warm and cold temperatures does not always elicit the same results. Furthermore, variable alterations can have different effects on specified tasks. This suggests that the design of thermal stimuli is highly dependent on what task result should be maximized.
\end{abstract}

\section{Author Keywords}

Audio; in-car; thermal.

\section{CCS Concepts \\ -Human-centered computing $\rightarrow$ Haptic devices;}

\section{INTRODUCTION}

Visual feedback from dashboards and infotainment systems is commonly used to inform the driver about the state of the car. However, such feedback competes for the driver's visual attention and can increase the likelihood of accidents due to distraction [9]. Therefore, it is important to explore alternative ways to reduce visual distraction and allow the driver to focus on looking at the road ahead. While audio feedback can be masked by in-car media, such as listening to music, haptic feedback can be used unobtrusively and be directed to the driver alone.

AutomotiveUI '18 10th International Conference on Automotive User Interfaces and Interactive Vehicular Applications, September 23-25, 2018, Toronto, ON, Canada

ACM ISBN 978-1-4503-2138-9.

DOI: $10.1145 / 3239060.3239062$
Many experiments have investigated the integration of haptic feedback into the car, mostly focusing on vibrotactile interaction. Tactile feedback was rated as mentally less demanding than the visual modality when presenting navigational cues with vibration motors in the seat [18]. The combination of tactile with other modalities has further been shown to decrease both reaction time [18, 16, 7, 14] and lane deviation [8]. This shows that the driver can react faster to input presented by the car with tactile feedback than visual alone and is less distracted from the driving task.

However, there are other aspects to cutaneous haptics than just vibration which could potentially be used to convey information. Research into thermal feedback has shown that the direction of change (hot or cold) can be distinguished with $94 \%$ accuracy and the subjective intensity with $73.1 \%$ accuracy, when presented on mobile phones [21]. This suggests that thermal feedback could be an effective way of conveying information to drivers. Additionally, thermal feedback can be unobtrusive [10]. This is a beneficial feature for feedback in a car, where sudden prompts can be distracting and therefore dangerous. Furthermore, thermal cues can elicit affective responses in different contexts $[12,15,22]$, presenting interesting and novel possibilities for in-vehicle interaction.

We conducted two driving simulator studies to investigate the effect of thermal interaction on drivers. Both utilized a lane change task and while the first was conducted to gain insight into drivers' reactions to this new feedback modality and compare these to audio feedback, the second investigated the influence of variable temperature changes on the lane change task.

\section{Contributions}

- We investigated driver reactions to the presentation of thermal feedback while driving;

- We found a $1.82 \mathrm{~s}$ increase in time needed to complete a lane change in the thermal condition (with a temperature change time of $2 \mathrm{~s}$ ) compared to audio but no difference in lane deviation performance;

- We examined the influence of variable changes of the thermal stimuli on the lane change task.

With these results as a baseline, more detailed and varied investigations into thermal in-car feedback can be conducted. 


\section{RELATED WORK}

Research into haptic feedback in vehicles usually focuses on the use of vibration, both in varying locations and for different purposes. Vibration is often used as feedback type because it is easy to implement. Its characteristics have been investigated for general use outside the car [4, 2] and for a wide range of uses, such as warning representation [6] and instructions [13]. It has been examined in most detail for mobile devices [1, 5, $3]$. In the car, haptic feedback is mostly used either to warn the driver or to give navigational instructions.

Kern et al. [8] investigated the benefits of vibration on the steering wheel in addition to auditory or visual cues when presenting navigational information in two experiments utilizing a lane change task. Their first experiment showed that many participants had problems to determine where exactly the vibration originated. They also could not easily interpret the instructions when presented without audio. Driving performance was worse and the participants stated that they preferred the audio-only or audio and tactile combination. As a result, they refined their apparatus and conducted a second experiment to test if adding vibrotactile to multimodal audio and visual cues would enhance performance. They found that the multimodal conditions including vibration resulted in better driving performance and the visual and tactile combination was preferred by the participants. This indicates that haptic feedback can enhance both the driving performance as well as the driving experience, especially when used in a multimodal setting. These findings were affirmed by Politis et al. [14].

Utilizing haptic shear feedback (moving the skin of the user) Medeiros-Ward et al. [11] presented a steering wheel with two tactors that moved the skin in the direction of the desired lane change, and then back after a short pause. The tactors were small and could be touched by a fingertip. The haptic feedback was tested against audio navigation. Even though the results for the single task modality were almost equivalent, there was a significant difference when a phone conversation was added as a secondary task. This led to a loss in navigation accuracy for the auditory but not the haptic modality. This indicates a robustness of tactile feedback in the presence of auditory distractions. These are common in the car, for example with phone calls, music and conversations with passengers, and means haptics might be a valid alternative feedback type to existing audio-based secondary tasks.

Van Erp and van Veen [18] built vibrators into a car seat and presented navigational cues through vibration on the specific side under the thigh. They used different patterns to indicate the distance to the turning point. They found a significant reduction in reaction times when combining vibration with visual presentation of navigation information, while the difference for visual-only to tactile-only was not significant. Mental workload was noticeably increased in the visual condition compared to both the tactile and multimodal cues. They tested participants under different workload conditions and testing the peripheral detection time by presenting randomly timed visual stimuli on the left visual periphery. Results showed a $10 \%$ increase in the peripheral detection time for the visual only navigation in the higher workload condition, while there were no significant differences for the tactile and multimodal feedback. The results showed that vibration could be especially beneficial for time sensitive warnings in a car.

These papers demonstrate the potential of tactile feedback for conveying information to drivers whilst minimising visual distraction. Combining tactile with other types of feedback was especially effective. We extend this work by investigating the use of thermal feedback in cars. This could provide an additional tactile channel for conveying information to drivers.

\section{Thermal Interaction}

To date thermal interaction has not been investigated in the incar environment. However, many cars do already use thermal hardware in the form of heated seats and steering wheels making it familiar to drivers. There have, however, been experiments studying thermal feedback in mobile devices. Wilson et al. [24] gave recommendations for the use of thermal interactions after a suite of studies, identifying the thenar location (base of thumb) as the most suitable feedback region, where both warm and cold stimuli were effectively perceivable. They also tested different rates of temperature change, finding $1 \% \mathrm{~s}$ and $3 \% \mathrm{~s}$ both equally detectable. $1 \% \mathrm{~s}$ was rated more comfortable by participants, but took longer to be recognized, while $3 \%$ was rated less comfortable but was detected faster. In another set of experiments [21], they asked participants to engage with four thermal icons, combining the two directions of temperature (warm and cold), two different rate of change $\left(1^{\circ} / \mathrm{s}\right.$ and $\left.3^{\circ} / \mathrm{s}\right)$ and two temperature change values $\left(3^{\circ}\right.$ and $\left.6^{\circ}\right)$, in outdoor mobile tasks. The results showed a moderate recognition rate of the complete thermal icons (64.4\%), while the features direction of change and rate of change both had higher recognition ( $94 \%$ and $73.1 \%$, respectively). These results are very promising. Mobile devices are used for a wide variety of tasks in unpredictable environments, conditions that have many similarities to the car.

With the goal to determine whether different people interpret thermal feedback in the same way in different contexts, Wilson et al. [22] prepared four scenarios and asked naive participants to attribute meanings to the thermal feedback presented. The tasks varied from using temperature to rate restaurants or bars, deciding whether an office was in use and how busy the occupant was, to determining when social media was last accessed. The participants consistently used warm stimuli to rate more positive experiences, physical presence, busyness and recent activity, with cooler stimuli less positive or less active. If this was the same in the car, then haptic feedback could potentially be used without extensive training.

Temperature has been used to provide navigation information. In an initial study Wettach et al. [19] used a mobile thermal device to help pedestrians walk towards their target by warming when they walked directly towards the end point, presenting five different temperatures. The participants could find their way to the destination, but criticized the lack of distance information. A similar approach was used by Tewell et al. [17] to assist navigation through a 2D maze. They presented constant feedback, warm if the participant was on the best path through the maze, which then cooled slowly when they left it. Compared to the control condition, in which no additional support 


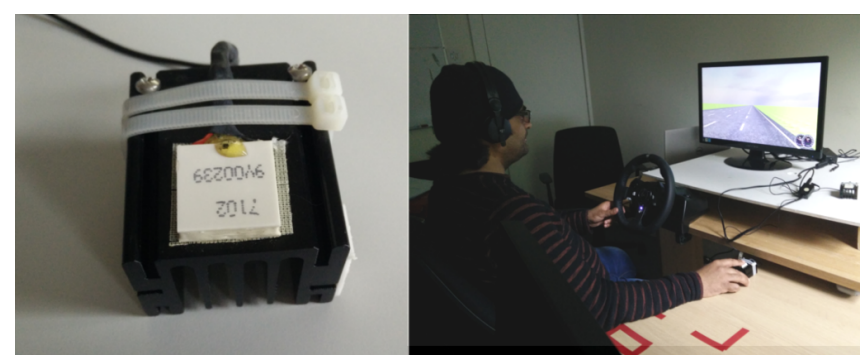

Figure 1. The Peltier device (left) and the experimental set-up (right).

was given, the number of moves decreased significantly with the thermal cues, but the speed through the maze was slower when presented with thermal feedback.

Combining ideas for in-car haptic interaction with the findings from thermal feedback research leads to a number of interesting questions. How can the most common types of feedback for drivers, like navigation cues and warnings, be presented with thermal feedback? Is thermal interaction distracting while driving? Is its use effective for navigation?

We conducted two exploratory experiments to begin answering these questions. The first investigated the implications of thermal feedback on the driving performance and the second explored the design space of thermal stimuli with a single device for in-car use.

\section{APPARATUS}

The same set-up was used in both experiments. It took place in a university room, in which the participants were seated in front of a 23.6-inch HannsG HL249 monitor. The driving simulation was implemented with OpenDS $3.5^{1}$ and the scene depicted a five-lane motorway. The car maintained a constant speed of $100 \mathrm{~km} / \mathrm{h}$ throughout all parts of the experiment. The study was operated from a DELL XPS 159550 laptop using Windows 10 with a Logitech G920 Driving Force steering wheel attached to it. Audio was presented through Sennheiser HD 25-1 II Basic Edition headphones, which were worn throughout the driving parts of the experiment. The $2 \times 2 \mathrm{~cm}$ Peltier element with an attached heat sink (see Figure 1 (left)) was designed and built by SAMH Engineering and was controlled via Bluetooth. The thermal stimuli were always presented on the right hand, while the participants drove with their left one. In the audio condition the participants were asked to place their right hand on the table. The complete set-up can be seen in Figure 1 (right). Both experiments were approved by the Ethics Committee of our institution.

\section{EXPERIMENT 1}

This first experiment was conducted to collect basic data about thermal interaction while driving. We were particularly interested in investigating the participants' performance of the driving task itself and their preference when compared to auditory feedback, as the most widely used non-visual feedback type in cars. The transmitted information communicated the direction of lane change.

\footnotetext{
${ }^{1}$ http://www.opends.eu
}

In accordance with the results of the first study described by Kern et al. [8] and due to the fact that thermal feedback may be new to the participants, we expected the distraction level to be higher and the driving performance thus to be lower than in the audio condition. On the other hand, the smooth and continuous change of temperature compared to the sudden and loud presentation of audio instructions should make the experience more pleasant and less disruptive for participants. Furthermore, as the detection time of thermal feedback is longer than for audio, a faster lane change in the auditory modality was expected. With children's games like "Hot or Cold" in mind, we expected more participants to choose to turn towards the direction of the warmed side of a steering wheel rather than towards the direction of the cooled one.

Therefore, the hypotheses for this study were:

Hypothesis 1: Thermal feedback will be more distracting than auditory;

Hypothesis 2: Thermal feedback will be rated as less disruptive and more pleasant than the audio feedback;

Hypothesis 3: The time taken to complete a lane change will be longer in the thermal condition;

Hypothesis 4: More participants will choose to turn towards the direction of the warm side of a thermal steering wheel.

\section{Design}

The experiment used a within-subjects design with the two conditions: auditory and thermal feedback. The direction of desired lane change was indicated by the direction of temperature in the thermal condition. The neutral temperature was set to $30^{\circ} \mathrm{C}$ and heated to $36^{\circ} \mathrm{C}$ when the participants should change one lane to the right, while it was cooled to $24^{\circ} \mathrm{C}$ for a change to the left. The temperature change occurred at a rate of $3 \%$, therefore needing 2 seconds to reach the desired temperature. This was then held for 8 seconds until the Peltiers were returned to the neutral temperature. The values for the temperature change extent and the rate of change were chosen in accordance with findings from studies by Wilson et al. [23, 21, 20, 24].

The lane change study consisted of two parts: in the first the participants were presented with the stimuli but did not change lanes; we wanted to see if stimuli presentation alone would influence lane deviation and driving behaviour. They were asked to report the recognized stimuli verbally back to the experimenter. In the audio condition, the participants were presented with either "Warm" or "Cold", uttered by a male synthesized voice. We decided on these words for the first part to not elicit a lane change from the driver, which might have been prompted by using the words "Right" and "Left". Furthermore, these words matched the thermal cues. Each participant was presented with 10 thermal and 10 audio stimuli in the corresponding condition. The lane deviation results would indicate the level of distraction posed by the two conditions. Deviation was calculated for four different time frames: 5 seconds before the stimulus presentation, 2 seconds of temperature change, 8 seconds of the constant temperature presentation and then finally the 2 second change back to the neutral temperature. These time frames would allow us to see 
if the different parts of the thermal stimulus had an impact on the level of distraction. The deviation before the stimulus was taken as a baseline. The Root Mean Square Error (RMSE) of the logged X-positions from zero were calculated for the time stages of each stimulus.

In the second part, participants were asked to actually turn when presented with the feedback, thus testing a more realistic driving context. The auditory cues here were "Right" and "Left". These instructions were chosen to mimic feedback already established in car navigation systems. The thermal stimuli were the same as in the first part. The time taken for the completion of the lane change and the number of correct lane changes was measured.

\section{Participants}

Fourteen right-handed participants ( 8 female) aged between 20 and 34 years $(M=26.36, S D=4.68)$ and mostly students took part in this study. They all held a valid driving licence and had driving experience between 1 and 17 years $(M=7.71$, $S D=4.92$ ). All reported at least corrected vision and no sensory impairments of the hands.

The participants reported their prior experience using a driving simulator on a scale from 1 (not at all) to 5 (very much) with a $M=2.00(S D=1.29)$ and experience using audio navigation with $M=2.93$ ( $S D=1.63)$. Thermal interaction was less known, with a range from 1 to 3 and $M=1.18(S D=0.54)$.

Each participant was offered $£ 6$ for their participation in this one-hour long study.

\section{Procedure}

After reading the information sheet and signing the consent form, participants were first given a chance to get used to the driving simulator. They could drive on the motorway until they felt comfortable. Afterwards the thermal interaction was presented and each temperature was shown to the participants. This was to ensure that the participants could feel both the temperature change as well as the return to neutral. When they felt confident about the thermal interaction, they started with the first part of the experiment, followed by the second part after a short break. The order of the conditions in both blocks was counterbalanced.

The participants filled in NASA TLX $^{2}$ questionnaires and questions about pleasantness after each condition in the second part. At the end of the experiment, each participant was also asked to rate the two conditions on a five-point Likert scale and asked to name their preference of turning direction presentation through temperature. For this they were presented with following question:

"Imagine you were presented with thermal feedback on the steering wheel for navigation purposes, where the devices of one side of the wheel will be warmed, while the other side will be cooled, how would you interact: If the right side of the steering wheel was warmed, while the left side was cooled, I would turn to the: left / right"

The results of this question would help design future navigation studies and give an indication about the generalizability

\footnotetext{
${ }^{2}$ https://ntrs.nasa.gov/archive/nasa/casi.ntrs.nasa.gov/20000021488.pdf
}

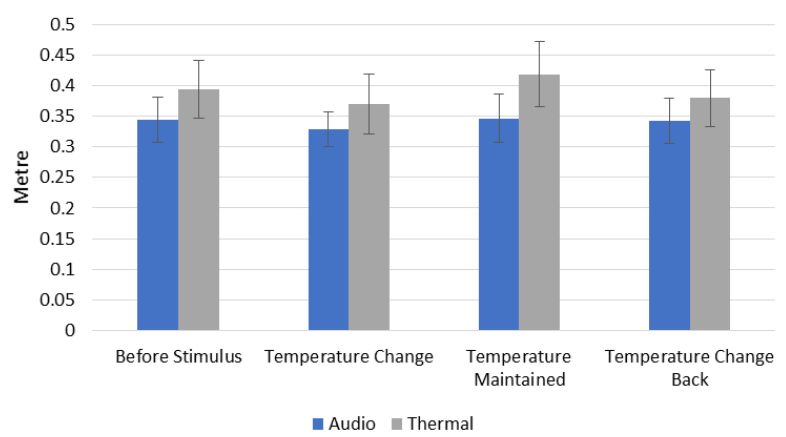

Figure 2. The lane deviation in the first part of the Lane Change Scenario was divided into several stages for comparison. The error bars on all bar charts show Standard Error.

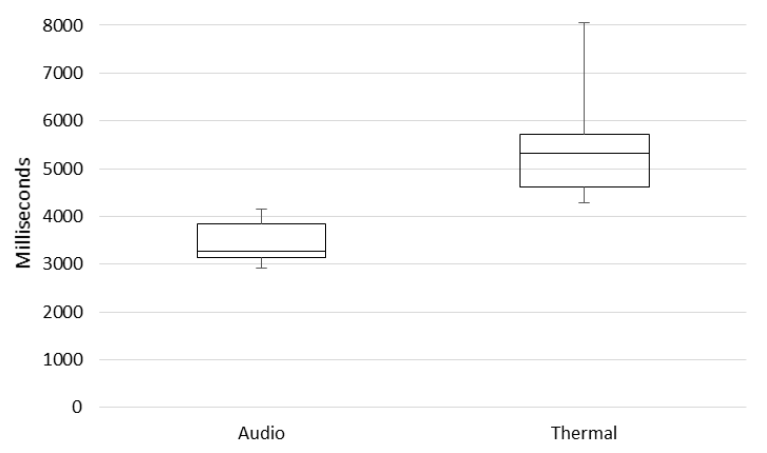

Figure 3. The time taken to complete the lane changes for the two conditions. The error bars show the minimum and maximum time, while the boxes visualize the first and third quartile around the median (line).

of the correlation between direction of temperature change and turning direction in a navigation task.

\section{Results}

Lane Deviation

The Root Mean Square Error (RMSE) of the lane deviation ranged between $0.33 \mathrm{~m}$ and $0.35 \mathrm{~m}$ in the audio and $0.37 \mathrm{~m}$ and 0.42 in the thermal condition, see Figure 2. Overall the lane deviation in the thermal condition was between $3.7 \mathrm{~cm}$ and $7.2 \mathrm{~cm}$ higher. It was not normally distributed and Wilcoxon tests showed no significance (before $Z=1.475, p=0.140$, temperature change $Z=0.471, p=0.638$, temperature maintained $Z=1.654, p=0.096$ and temperature change back $Z=0.722$, $p=0.470)$.

\section{Time to Complete a Lane Change}

Mean time to complete a lane change was 4368.1 milliseconds (Figure 3). This was higher for thermal $(M=5.28 \mathrm{~s}, S D=0.75)$ than audio $(M=3.46 \mathrm{~s}, S D=0.42)$, with a difference of 1.82 seconds. A paired t-test found this difference significant: $t(14)$ $=12.84, \mathrm{p}<0.001$.

\section{Stimulus Recognition}

The recognition accuracy of the feedback cues in the first part of the study was $100 \%$ for the audio and $97.14 \%$ for the thermal condition; 4 out of 140 thermal cues were incorrectly 


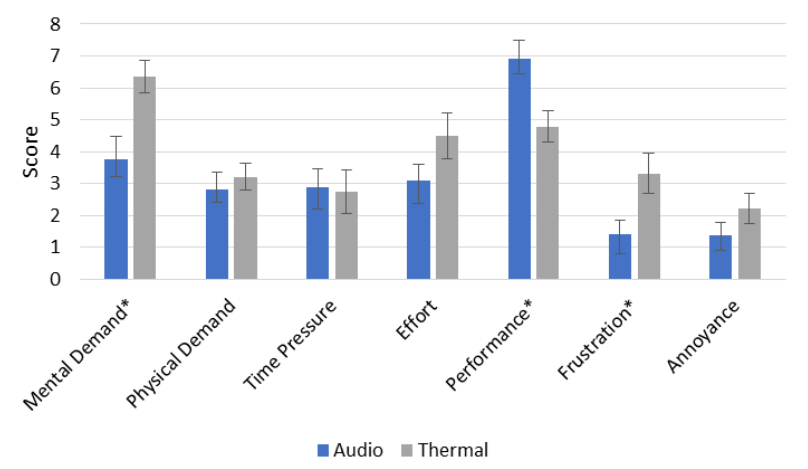

Figure 4. The mean values of the NASA TLX questionnaire for the Lane Change Scenario. Significant differences are marked with an asterisk.

recognised. Three of these were missed and in one case the temperature direction was identified wrongly. However, 25 additional stimuli were identified. These were mostly misinterpretations of the temperature change back to neutral; participants mistook the change back to neutral as a new stimulus, a false positive.

In the second part, the recognition error rate was $11.43 \%$ in the thermal condition (16 missed stimuli). The number of false positive stimuli went down to a total of 8 . These occurred for changes back to the neutral condition both from cold (6 times) and warm (2). In the audio condition all stimuli were recognized correctly.

\section{Qualitative Rating}

The results of the NASA TLX ratings can be seen in Figure 4. A Wilcoxon test showed a significant difference between the two feedback types for overall workload $(Z=2.605$, $p=0.009$ ) with a median of 19 for the audio condition and 27 for the thermal condition. A more detailed comparison showed significant differences for mental demand, performance and frustration, with $(Z=2.708, p=0.007$, median (audio) $=3$, median(thermal $)=7),(Z=2.395, p=0.017$, median(audio $)=8$, median (thermal $)=5$ and $(Z=2.273, p=0.023$, median (audio) $=1.25$, median (thermal) $=3.75$, respectively.

Subjective responses to the conditions were determined through four questions, rated on five-point Likert scales. Participants were asked to rate how "pleasant" and "comfortable" the feedback felt (positive aspects), and how "disruptive" and "complicated" (negative aspects). The results can be seen in Figure 5. The positive aspects showed no significant difference (pleasantness: $Z=1.742, p=0.082$, median $($ audio $)=3$, median $($ thermal $)=3$; comfort: $Z=0.957$, $p=0.339$, median $($ audio $)=3.5$, median $($ thermal $)=3.25$ ) for the feedback types. The negative aspects did for complexity $(Z=3.075, p=0.002$, median (audio $)=1$, median $($ thermal $)=3)$ but not disruptiveness $(Z=1.444, p=0.149$, median(audio $)=$ 1.5 , median $($ thermal $)=2$ ).

\section{Turning Direction}

When asked for their preference regarding the mapping of turning direction to temperature, 14 participants chose "right",

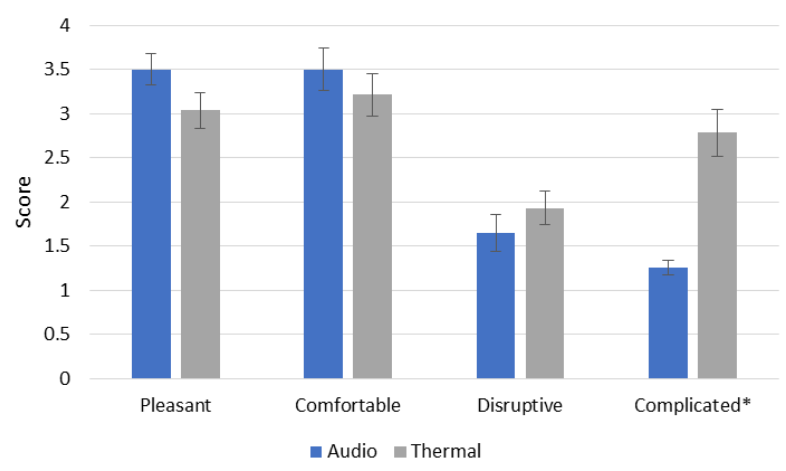

Figure 5. The mean ratings for pleasantness, comfort, disruption and complexity for the Lane Change Task. Significant differences are marked with an asterisk.

which corresponds to the warm side, while only 1 chose "left", the cold side.

\section{Discussion}

This study was designed to gain some general information and first impressions on thermal interaction while driving. There was no significant difference in lane deviation between the thermal and the audio condition, when the stimuli were presented but no lane change occurred. This suggests that use of thermal feedback during driving was no more distracting than audio, which does not corroborate Hypothesis 1. However, based on the trends shown in Figure 2, Hypothesis 1 might be supported if a larger sample was tested.

The comparison of the times used to complete the lane changes showed that, on average, an additional 1.82 seconds was needed to complete lane changes in the thermal condition, which supports Hypothesis 3.

However, Hypothesis 2 regarding the higher rating for pleasantness and lower rating for disruptiveness was not supported. The thermal feedback was rated as more complex and the mental demand and frustration described by the participants were higher. Audio feedback was named as being more familiar and therefore easier to utilize, which was given as a reason for rating the thermal feedback more negatively. Furthermore, an additional level of mapping was needed in the thermal condition: "warm" had to be translated to "right".

Participants reported that they sometimes had difficulties distinguishing between warm and cold at first. It took them some time to be sure about the temperature direction.

Some participants had problems understanding if the temperature change back to neutral was a new stimulus or not. This suggests that a different stimulus representation with less extent of change or less distinguishable transition to the neutral temperature should be investigated. A reduction in stimulus length could also change the perception, as the skin might be less adapted to the stimulus temperature when it is returned to the neutral temperature.

Most participants preferred the mapping of warmth to the turning direction. Hypothesis 4 is therefore proven and corresponds with the mapping in related work $[19,17]$. The results 
suggest that thermal feedback can be used for applications that are not time urgent, because it needs more time to be recognized.

A second experiment was prepared to investigate if a change of the stimulus design could reduce the false positives that occurred during the return to the neutral temperature, without increasing the error rate.

\section{EXPERIMENT 2}

For this experiment, the feedback contained again both temperature increases and decreases, indicating the direction of the desired lane change. However, combinations with several stimuli lengths were employed, shorter than the ones used in the first experiment. Additionally, two types of slower rates of change for the return to the neutral temperature were used, as well as two extents of temperature change. These changes were made to support the participants' ability to distinguish between the stimulus itself and the return to the neutral temperature afterwards. These changes should cause no negative effect on the recognition rate. We expected that the parameters which made the return to the neutral temperature the least noticeable would decrease the number of false positives. Furthermore, a shorter presentation time would clarify the connection of the change back to neutral to the stimulus.

The hypotheses of this study therefore were:

Hypothesis 1: The slowest return to the neutral temperature will have the smallest number of false positive lane changes at the return to the neutral temperature;

Hypothesis 2: A shorter presentation time will have the smallest number of false positive lane changes at the return to the neutral temperature;

Hypothesis 3: The higher extent of temperature change will have the better recognition rate.

\section{Design}

The study employed a within-subject design with 4 independent variables: direction of temperature change $(D I R)$, length of temperature presentation $(L E N)$, rate of change at the return to the neutral temperature $(R O C)$ and extent of temperature change $(E X T)$.

The neutral temperature was again set at $30^{\circ} \mathrm{C}$. The thermal device was either cooled or warmed $(D I R)$ for $3^{\circ} \mathrm{C}$ or $6{ }^{\circ} \mathrm{C}$ at $3^{\circ} \mathrm{C} / \mathrm{s}$. The direction of the change defined the direction of the lane change as in the first experiment: cold indicated the left side, while warm represented a change towards the right lane. The temperature was presented for 0,3 or 6 seconds $(L E N)$ after the target temperature was reached. Two rates of change (ROC) were employed for the return back to neutral. Two rates of change $(R O C)$ were employed for the return back to neutral including $1^{\circ} \mathrm{C} / \mathrm{s}$ and $0.5^{\circ} \mathrm{C} / \mathrm{s}$. Because the used hardware only allowed for a rate of change of either $3^{\circ} \mathrm{C} / \mathrm{s}$ or $1{ }^{\circ} \mathrm{C} / \mathrm{s}$, the $0.5^{\circ} \mathrm{C} / \mathrm{s}$ change rate was simulated by increasing or decreasing the temperature at $1^{\circ} \mathrm{C} / \mathrm{s}$ for one second, followed by holding the temperature for one second. The stimuli were labelled DIR-LEN-EXT-ROC. For example, a cool temperature change of $3^{\circ} \mathrm{C}$, which was held for 6 seconds and returned to the neutral temperature at an angled rate of change of the simulated $0.5^{\circ} \mathrm{C} / \mathrm{s}$ was labelled $c-6-3-a$. Each combination of these variables, 24 in total, was presented 3 times to each participant, resulting in 72 stimuli for each participant. These were randomly ordered and divided into 8 blocks.

\section{Participants}

Sixteen right-handed and newly recruited participants ( 8 female) between 19 and 35 years $(M=25.88, S D=5.06)$ completed this experiment. They were mostly students and held a valid driving license. Their driving experience ranged between 0 and 17 years $(M=5.28, S D=5.20)$. They all reported at least corrected vision and no sensory impairments in the fingers. The participants rated their prior experience with a driving simulator and thermal feedback on a Likert scale (1 (none) to 5 (much)). The familiarity with a driving simulator ranged between 1 and $5(M=3.25, S D=1.29)$, with thermal feedback between 1 and $4(M=2.00, S D=1.32)$. The study took about one hour and each participant was paid $£ 6$.

\section{Procedure}

The participants were presented with an information sheet and asked to sign the consent form. Afterwards they were given the chance to familiarize themselves with the driving simulator by driving along the motorway until they felt comfortable. The participants were then introduced to the different temperature changes. Both temperature extends in both directions were shown and explained to the participants, showing the different stimuli types tested in the experiment. The driving task itself consisted of 8 blocks with 9 stimuli each. The participants could take breaks between the blocks, if needed.

At the end of the experiment the participants filled in questionnaires, capturing demographic data.

\section{Results}

The results for both the lane changes back to neutral and recognition were calculated separately for warm and cold.

\section{Lane Changes at Return to Neutral Temperature}

The evaluation of the warm changes with a repeated measures ANOVA showed no significance for $\operatorname{ROC}(F(1,15)=0.000$, $p=1.000)$ and EXT $(F=(1,15)=1.200, p=0.291)$. For $L E N$ the sphericity was violated and with GreenhouseGeisser corrections showed no significant difference $(F=(1.460,21.903)=2.416, p=0.125)$.

The cold temperature transitions were also evaluated with a repeated measures ANOVA and showed no significant differences for $\operatorname{EXT}(F(1,15)=0.345, p=0.566)$, $\operatorname{ROC}(F(1,15)=0.394, p=0.539)$ and $\operatorname{LEN}(F(2,30)=1.923$, $p=0.164$ ). Figure 6 illustrates the percentages of erroneous changes for each parameter. Even though there are no statistically significant results, some trends can be seen. The $L E N$ of 6 seconds lead to more lane changes for both warm and cold stimuli. Additionally, EXT seems to impact the changes in opposite ways: while a higher extent increases lane changes for warm temperatures, it seems to reduce it for cold temperatures. $R O C$ only seems to have a small impact on cold stimuli, not warm. A more extensive study has to be conducted to see if these trends influence the results consistently. Figure 7 shows the false positive lane changes for each stimulus. 


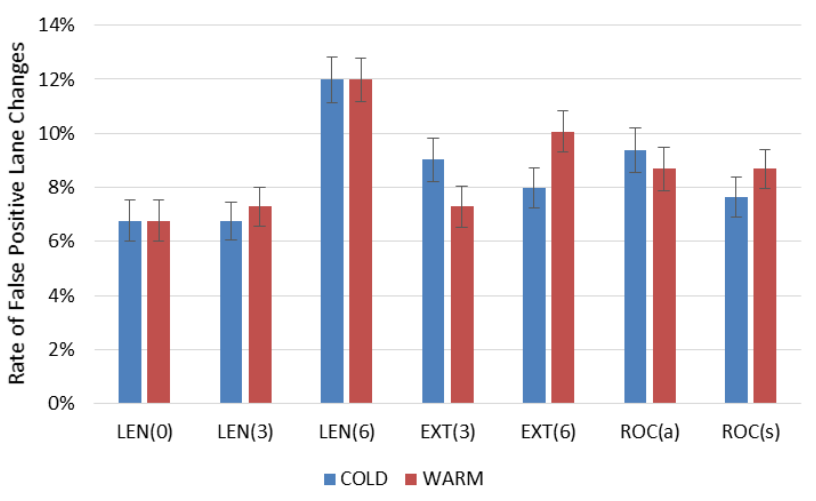

Figure 6. Percentage of the means of false positive lane changes at the return to the neutral temperature for the different parameters for warm and cold temperature changes.

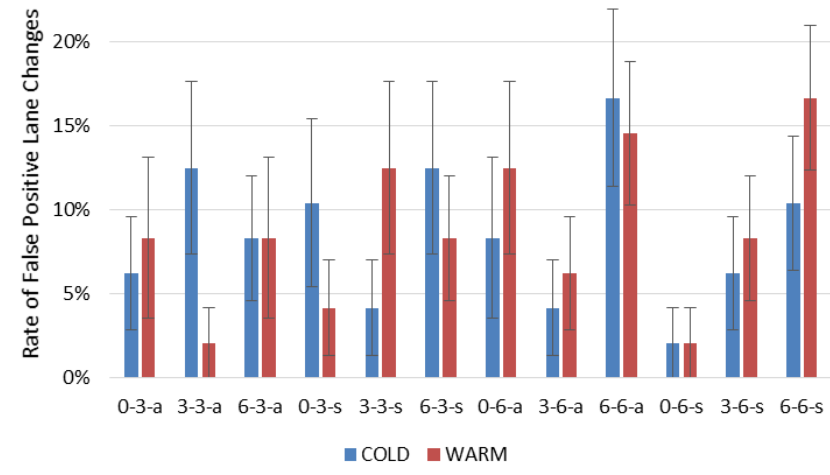

Figure 7. Percentage of the means of false positive lane changes at the return to the neutral temperature for the different stimuli combinations (LEN-EXT-ROC) for the warm and cold temperature changes.

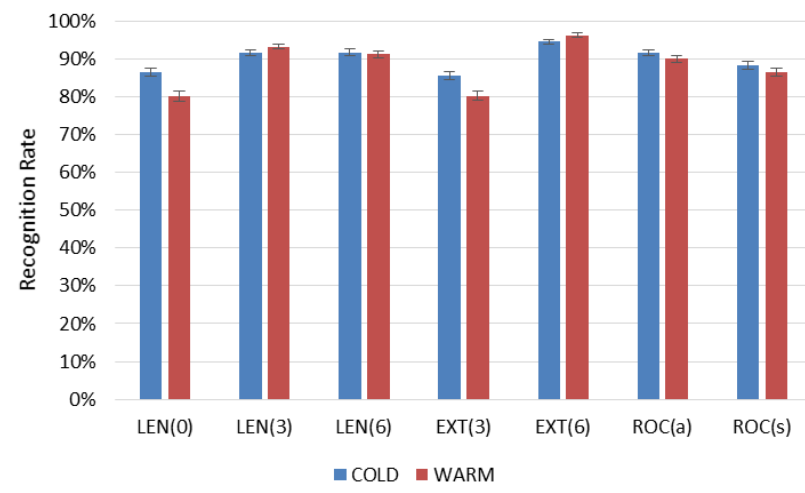

Figure 8. Recognition Rate of the second experiment for the parameters, both warm and cold temperature changes.

\section{Recognition}

A repeated measures ANOVA was employed to evaluate the warm stimuli for recognition. Sphericity was violated for LEN and showed significant differences with GreenhouseGeisser corrections $(F(1.413,21.197)=10.748, p=0.002)$. Post hoc tests were adjusted with Bonferroni corrections and show significant differences between the presentation lengths of 0 and 3 seconds $(p=0.011)$ and 0 and 6 seconds $(p=0.006)$, but not between 3 and 6 seconds $(p=1.000)$. The recognition rate was significantly lower when the temperature returned to neutral immediately (length of $0 \mathrm{~s}$ ) (154 of 192 correct recognitions overall) compared to the other two lengths (179 for $3 \mathrm{~s}$ and 175 for $6 \mathrm{~s})$. EXT showed significant results as well $(F(1,15)=23.134, p<0.0005)$, where the temperature change of $6^{\circ} \mathrm{C}$ had a better recognition rate (277 out of 288) than $3^{\circ} \mathrm{C}(231) . R O C$ did not show significant differences $(F(1,15)=2.358, p=0.145)$.

The only significant difference for the cold stimuli that were shown by the repeated measures ANOVA was found for $\operatorname{EXT}(F(1,15)=8.366, p=0.011)$. The higher extent of $6^{\circ} \mathrm{C}$ resulted in a better recognition rate (272 out of 288) than $3^{\circ} \mathrm{C}(246)$. Both $\operatorname{LEN}(F(2,30)=1.344, p=0.276)$ and $R O C$ $(F(1,15)=1.471, p=0.244)$ showed no significant differences. See the recognition rates for the parameters in Figure 8 and for each individual stimulus in Figure 9.

\section{Time to Complete a Lane Change}

The mean time it took the participants to complete a lane change can be seen in Figure 10 for each stimuli. The overall mean time for cold stimuli was $5.11 \mathrm{~s}(S D=0.45)$ and for warm stimuli $5.17 \mathrm{~s}(S D=0.14)$. The mean times were not normally distributed and a Wilcoxon test showed no significance for temperature direction on the time to complete lane change $(Z=1.241, p=0.215)$.

\section{Discussion}

The investigation of the false positive identification of transitions back to neutral showed no statistical significant results. However, some trends could be identified, which should be monitored in future studies: the extent of temperature change might lead to a higher number of lane changes for warm 


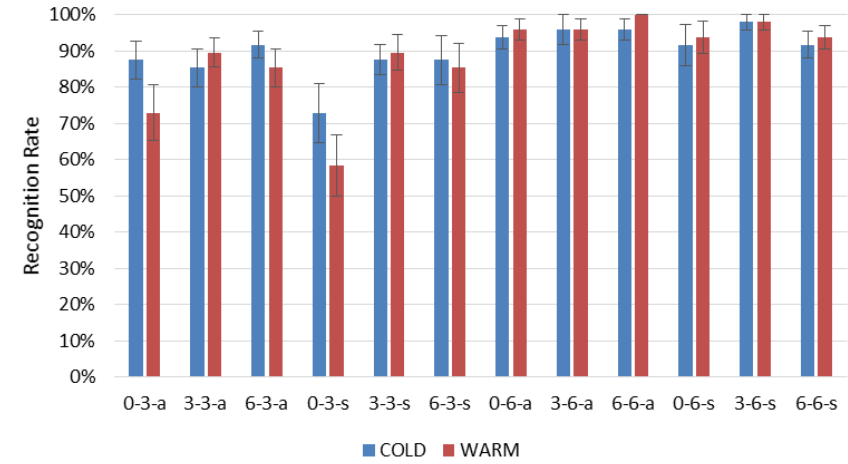

Figure 9. Recognition Rate of the second experiment for all stimuli variations (LEN-EXT-ROC), both warm and cold temperature changes.

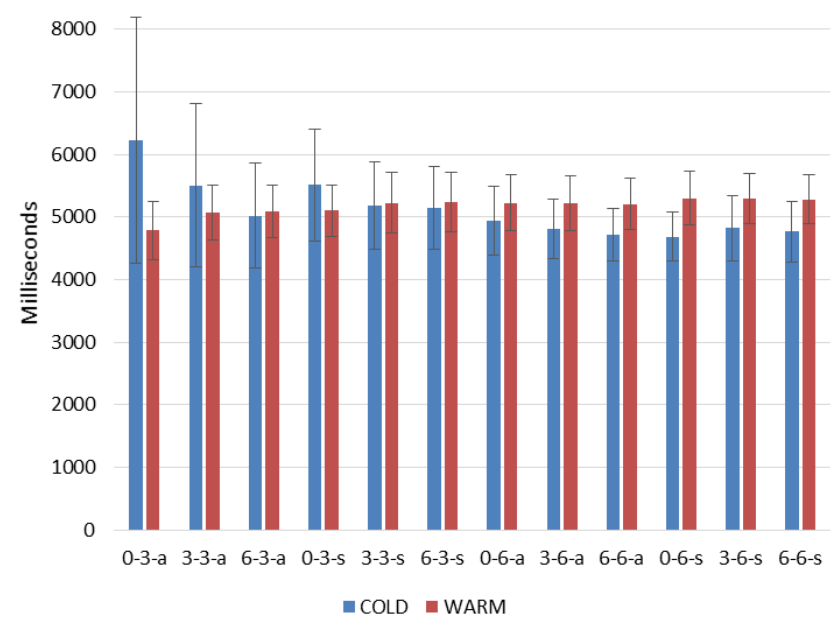

Figure 10. Mean times to completed lane change for all stimuli combinations (LEN-EXT-ROC) for the warm and cold temperature changes. temperatures, while it seems to be the opposite way around for cold temperatures, where the lesser extent seems to have elicited more changes back. In the case of cold temperature changes an additional correlation with the rate of change might exist: the slowest rate of change seems to lead to more lane changes. Therefore, both Hypothesis 1 and 2 are not supported. Additionally, both temperature changes seem to have a higher number of false positive changes for longer stimuli.

The correct recognition of stimuli was aided by a higher extent of temperature change for both directions, as predicted in $\mathrm{Hy}$ pothesis 3. Additionally, in the case of increasing temperature, the length of stimulus representation influenced the recognition rate, but only if the temperature was not held constant at all. There was only one stimulus with $100 \%$ recognition rate: w-6-6-a. Unfortunately, the number of false positives was one of the highest for this stimulus.

These differences in how the results are affected for the two directions of temperature show that a direct mirroring of stimuli design might not work for all use cases. Additionally, the choice of stimulus features depends highly on the prioritized result: should the design aim for a high recognition rate or try to minimize false positive lane changes?

\section{CONCLUSION AND FUTURE WORK}

These two studies give first insights into the performance of thermal feedback during driving. More experiments have to be conducted to confirm these findings, as the sample size was small and limited to right-handed participants. A more realistic setting, allowing the participants to drive with two hands on the steering wheel and in a more car-like environment, might influence the results as well. Nonetheless, these experiments highlight some important features and challenges for thermal interaction during driving.

The distraction level of thermal feedback did not show a significant difference compared to audio feedback. The lane change in the thermal condition took under 5.3 seconds in both studies, less than 2 seconds longer than the audio condition. Participants rated thermal feedback as less pleasant than audio feedback. This rating could be influenced by the higher familiarity with audio feedback and the additional level of mapping needed in the thermal condition. Audio navigation systems are widely used in modern cars and most participants had some experience with their use. A comparison with another less familiar feedback type, such as vibrotactile feedback, could give more insightful results. This will be investigated in future work, along the use of thermal feedback for warnings, as these could benefit from the intrinsic impression of urgency and danger associated with hot temperatures. As the identification of thermal feedback takes time, the time-urgency of these warnings has to be carefully considered and investigated. Thermal feedback could be used for notifications of a less urgent manner, where vibration would be unnecessarily attention grabbing, as for example as a notification when fuel is running low, or for foreseeable events, such as turning points in navigation scenarios.

The broader investigation of different features of the thermal feedback showed that a change in design variables seems to not always influence the outcome in the same way for warm and 
cold temperatures: while a higher extent of warm temperature changes seems to lead to a higher number of false positive lane changes back at the return to the neutral temperature, whereas it seems to be the other way around for cold temperatures. These findings describe observed trends and were not backed up by statistical significance. Further investigations should keep these in mind, however, and monitor if these observation are consistent.

Furthermore, which variables influenced the recognition rate and the changes back varied and they were not identical for warming and cooling. There was no combination that eliminated the false positive lane changes at the return to the neutral temperature completely and, furthermore, no combination achieved both a minimal number of changes back and a good recognition rate together. Therefore, the design of the thermal stimulus is highly dependent on the prioritized feature. Conveying direction with thermal interaction without additional information does not seem to be feasible. Spatial information, given by the location of the thermal feedback, could aid the situation. This feedback could be given on the steering wheel or even the seat or seatbelt. Considering the distribution of temperature adjustable seats in cars, this kind of feedback could easily be implemented. A car provides the best possible environment for thermal feedback, as it is well defined, the driver's position is well known as is the in-car temperature. Additional installation of sensors could make thermal feedback even more adjustable. At the moment most warming steering wheels cannot cool, but as thermal comfort becomes more and more important, fully temperature adjustable steering wheels will be mounted in cars, completely eliminating any concerns about unwanted variations in temperature. To keep thermal comfort for the drivers, we envision thermal feedback to be adaptable, so drivers can individually set it according to their preference.

\section{ACKNOWLEDGEMENTS}

This research is is supported by an EPSRC iCASE Award and Jaguar Land Rover (Award Number EP/P510506/1).

\section{REFERENCES}

1. Jason Alexander, Mark T Marshall, and Sriram Subramanian. 2011. Adding haptic feedback to mobile tv. Proceedings of the 2011 annual conference extended abstracts on Human factors in computing systems - CHI EA'11 (2011), 1975. DOI :

http://dx.doi .org/10.1145/1979742 . 1979899

2. Stephen Brewster and Lorna Brown. 2004. Tactons: structured tactile messages for non-visual information display. AUIC'04 Proceedings of the fifth conference on Australasian user interface (2004), 15-23.

3. Stephen Brewster, Faraz Chohan, and Lorna Brown. 2007. Tactile feedback for mobile interactions. Proceedings of the SIGCHI conference on Human factors in computing systems - CHI 'O7 July (2007), 159. DOI : http://dx.doi.org/10.1145/1240624.1240649

4. Lorna Brown, Stephen Brewster, and Helen Purchase. 2005. A First Investigation into the Effectiveness of Tactons. (2005). http://www.dcs.gla.ac.uk/
5. Lorna Brown, Stephen Brewster, and Helen Purchase. 2006. Multidimensional Tactons for Non-Visual Information Presentation in Mobile Devices. Proceedings of the 8th conference on Human-computer interaction with mobile devices and services - MobileHCI 'O6 159, September (2006), 231-238. DOI: http://dx.doi.org/10.1145/1152215.1152265

6. Lorna M. Brown and Topi Kaaresoja. 2006. Feel who's talking: using tactons for mobile phone alerts. $\mathrm{CHI}$ 'O6 extended abstracts on Human factors in computing systems - CHI EA '06 (2006), 604. DOI : http://dx.doi.org/10.1145/1125451.1125577

7. Cristy Ho, Nick Reed, and Charles Spence. 2007. Multisensory in-Car Warning Signals for Collision Avoidance. Human Factors: The Journal of the Human Factors and Ergonomics Society 49, 6 (2007), 1107-1114. DOI : http://dx.doi .org/10.1518/001872007X249965.

8. Dagmar Kern, Paul Marshall, Eva Hornecker, Yvonne Rogers, and Albrecht Schmidt. 2009. Enhancing navigation information with tactile output embedded into the steering wheel. Lecture Notes in Computer Science (including subseries Lecture Notes in Artificial Intelligence and Lecture Notes in Bioinformatics) 5538 LNCS (2009), 42-58. DOI : http://dx.doi .org/10.1007/978-3-642-01516-8_5

9. S. G. Klauer, T. a. Dingus, V. L. Neale, J. D. Sudweeks, and D. J. Ramsey. 2006. The Impact of Driver Inattention On Near Crash/Crash Risk: An Analysis Using the 100-Car Naturalistic Driving Study Data. Analysis April (2006), 226. DOI : http://dx.doi .org/DOTHS810594

10. Wonjun Lee and Youn-kyung Lim. 2010. Thermo-message: exploring the potential of heat as a modality of peripheral expression. CHI'10 Extended Abstracts on Human Factors in ... (2010), 4231-4236. DOI : http://dx.doi.org/10.1145/1753846.1754131

11. Nathan Medeiros-Ward, Joel M. Cooper, Andrew J. Doxon, David L. Strayer, and William R. Provancher. 2010. Bypassing the Bottleneck: The Advantage of Fingertip Shear Feedback for Navigational Cues. (2010), 1650-1654. DOI :

http://dx.doi.org/10.1177/154193120104500402

12. Mutsuhiro Nakashige, Minoru Kobayashi, Yuriko Suzuki, Hidekazu Tamaki, and Suguru Higashino. 2009. "Hiya-Atsu" media. Proceedings of the 27th international conference extended abstracts on Human factors in computing systems - CHI EA 'O9 (2009), 3181. DOI : http://dx.doi.org/10.1145/1520340.1520453

13. Martin Pielot, Benjamin Poppinga, and Susanne Boll. 2010. PocketNavigator: Vibro-tactile waypoint navigation for everyday mobile devices. MobileHCI 2010 (2010), 423-426. DOI: http://dx.doi.org/10.1145/1851600.1851696

14. Ioannis Politis, Stephen Brewster, and Frank Pollick. 2014. Evaluating Multimodal Driver Displays under Varying Situational Urgency. (2014). DOI : http://dx.doi.org/10.1145/2556288.2556988 
15. Katri Salminen, Veikko Surakka, Jukka Raisamo, Jani Lylykangas, Johannes Pystynen, Roope Raisamo, Kalle Mäkelä, and Teemu Ahmaniemi. 2011. Emotional responses to thermal stimuli. Proceedings of the 13th international conference on multimodal interfaces - ICMI '11 (2011), 193-196. DOI :

http://dx.doi.org/10.1145/2070481.2070513

16. Keisuke Suzuki and Håkan Jansson. 2003. An analysis of driver's steering behaviour during auditory or haptic warnings for the designing of lane departure warning system. JSAE Review 24, 1 (2003), 65-70. DOI : http://dx.doi .org/10.1016/S0389-4304(02)00247-3

17. Jordan Tewell, Jon Bird, and George R. Buchanan. 2017. Heat-Nav. Proceedings of the 2017 CHI Conference on Human Factors in Computing Systems - CHI '17 (2017), 1131-1135. DOI :

http://dx.doi.org/10.1145/3025453.3025965

18. Jan B.F. Van Erp and Hendrik A H C Van Veen. 2004. Vibrotactile in-vehicle navigation system. Transportation Research Part F: Traffic Psychology and Behaviour 7, 4-5 (2004), 247-256. DOI : http://dx.doi.org/10.1016/j.trf.2004.09.003

19. Reto Wettach, Christian Behrens, Adam Danielsson, and Thomas Ness. 2007. A thermal information display for mobile applications. Proceedings of the 9th international conference on Human computer interaction with mobile devices and services - MobileHCI '07 (2007), 182-185. DOI : http://dx.doi.org/10.1145/1377999.1378004

20. Graham Wilson, Stephen Brewster, Martin Halvey, and Stephen Hughes. 2012. Thermal Icons: Evaluating
Structured Thermal Feedback for Mobile Interaction. Proceedings of the 14th international conference on Human-computer interaction with mobile devices and services - MobileHCI'12 (2012), 309-312. DOI : http://dx.doi.org/10.1145/2371574.2371621

21. Graham Wilson, Stephen Brewster, Martin Halvey, and Stephen Hughes. 2014. Thermal feedback identification in a mobile environment . In : 8th International Workshop on Haptic and Audio Interaction Design, 18-19 Apr 2013 , Daejeon, South Korea . Copyright (C) 2013 Springer-Verlag Berlin Heidelberg A copy can be downloaded for per October (2014), 18-19. https://doi .org/10.1007/978-3-642-41068-0

22. Graham Wilson, Gavin Davidson, and Stephen Brewster. 2015. In the Heat of the Moment: Subjective Interpretations of Thermal Feedback During Interaction. (2015), 18-23.

http://doi . acm.org/10.1145/2702123.2702219

23. Graham Wilson, Dobromir Dobrev, and Stephen A. Brewster. 2016. Hot Under the Collar: Mapping Thermal Feedback to Dimensional Models of Emotion.

Proceedings of the 2016 CHI Conference on Human Factors in Computing Systems (2016), 4838-4849. DOI : http://dx.doi.org/10.1145/2858036.2858205

24. Graham Wilson, M. Halvey, Stephen Brewster, and S.a. Hughes. 2011. Some Like it Hot: Thermal Feedback for Mobile Devices. Proceedings of the SIGCHI conference on Human Factors in Computing Systems - CHI' 11 (2011), 2555-2564. DOI : http://dx.doi.org/10.1145/1978942 .1979316 